\title{
Adaptive Framework for Energy Efficiency and User Satisfaction
}

\author{
Amol Gaikwad ${ }^{1}$, Ketan Patil ${ }^{2}$, Kedar Kerure ${ }^{3}$, Abhijit Rokade ${ }^{4}$ \\ Student, Computer, D.Y.P.I.E.T, Pune, India ${ }^{1,2,3,4}$
}

\begin{abstract}
Reducing energy demand in the residential and industrial sectors is an important challenge worldwide. In particular, lights account for a great portion of total energy consumption, and unfortunately a huge amount of this energy is wasted. Light emitting diode (LED) lights are being used to light offices, houses and building facilities more efficiently than traditional lights. Moreover, the light control systems are introduced to current markets, because the installed lighting systems are out dated and energy inefficient. However, due to high costs, installation issues, and difficulty of maintenance; existing light control systems are not successfully applied to home, office, and industrial buildings. In this system low cost, wireless, easy to install, adaptable, and smart LED lighting system to automatically adjust the light intensity to save energy and maintaining user satisfaction and also we control the light on the bases of Timing Control Android Application. The system combines motion sensors and light sensors in a low-power wireless solutions using wireless communication. Characterization of a commercial LED panel was performed to evaluate the benefit of dimming for this light technology. Measurements of total power consumption over a continuous six months period (winter to summer) of a busy office were acquired to verify the performance and the power savings across several weather conditions scenarios. This system reduces total power consumption. Saving energy has become one of the most important issues these days. The most waste of energy is caused by the inefficient use of the consumer electronics. Particularly, a light accounts for a great part of the total energy consumption. Various light control systems are introduced in current markets, because the installed lighting systems are outdated and energy-inefficient. However, due to architectural limitations, the existing light control systems cannot be successfully applied to home and office buildings. Therefore, this system, an intelligent household LED lighting system considering energy efficiency and user satisfaction is useful. This system utilizes multi sensors and wireless communication technology in order to control an LED light according to the user's state and the surroundings. This LED lighting system can autonomously adjust the minimum light intensity value to enhance both energy efficiency and user satisfaction. This LED lighting system reduces total power consumption to a great extent. However, since the existing lighting control systems can support only simple on/off or dimming control according to user movement or brightness of surroundings, it is hard to be applied to complex environments such as house or office. The complex environment means that there is a variety of control requirements, because of the presence of a variety of users. Because of this limitation of existing systems, they are mostly installed in the places such as the front door or the hallway. Furthermore, since the existing systems without considering user satisfaction, it is not appropriate to the places such as house and office where user satisfaction is more crucial factor than cost benefits due to energy saving; thus a new intelligent lighting control system should consider both energy efficiency and user satisfaction. In addition in this system we are adding power consumption for computers moreover we also control fans and saving lots of energy. Finally we monitor all the consumption of energy through Web Page. To implement this system we will require different electronic components like PIR,R-Pi 3,LDR,DHT11,Relay circuits, RTC etc.
\end{abstract}

Keywords: LED (Light Emitting Diode), PIR (Pyroelectric Infrared Sensor), DHT11 (Digital Humidity \& Temperature Sensor), RTC (Real Time Clock), LDR (Light Dependent Resistor).

\section{INTRODUCTION}

Energy saving and environmental friendliness/ awareness is a hot topic in current research. In fact, Carbon dioxide (CO2) emissions are strongly associated with energy consumption, these originated from the combustion of hydrocarbons (oil, natural gas and coal) either directly burned (transport and heating) or for generation of electricity in power plants. Lighting systems are a major source of electricity consumption in the world. Particularly, a light accounts for a great part of the total energy consumption. Various light control systems are introduced in current markets, because the installed lighting systems are outdated and energy-inefficient.
However, due to architectural limitations, the existing light control systems cannot be successfully applied to home and office buildings. Therefore, this system, an intelligent household LED lighting system considering energy efficiency and user satisfaction is useful. This system utilizes multi sensors and wireless communication technology in order to control an LED light according to the user's state and the surroundings. This LED lighting system can autonomously adjust the minimum light intensity value to enhance both energy efficiency and user satisfaction. This LED lighting system reduces total power consumption to a great extent. On the technological side, 
Light Emitting Diode (LED) is rapidly becoming a commonly used solid-state light source technology in general lighting applications. This is due to its longer lifetime, reduced power consumption, and having no poison mercury content compared with the conventional fluorescent lamps.

In addition, dimming control is often needed to regulate lighting levels for individual human needs or preferences as well as to achieve energy savings. Novel driver systems are improving the dimmable features to achieve this goal and are increasingly commercially available. This new technology is boosting interest in controlling the light to reduce power consumption. The market for lighting controls in residential and commercial buildings has entered a period of dramatic transformation. The demand for both wireless and local controls, such as object detection sensor and light sensors, temperature and humidity sensor and the adoption rate of the LED lighting systems begins to climb as well. With the advance of wireless sensor network (WSN) technology etc. It is now easier than ever to monitor and control houses, offices and industrial buildings. WSN is the backbone of a large variety of cyber-physical systems (CPS) applications in environmental monitoring, healthcare, security etc.

\section{RELATED WORK}

Research on monitoring, control and energy efficiency in the lighting domain has been prolific in recent years; with a variety of solutions and techniques proposed. The two main approaches are given by wired and wireless systems. Wired controllable lighting systems can measure the artificial and daylight illumination through the use of sensors in a controller area network or a set of data logger devices to modify the light intensity and hence its energy consumption. However, due to the presence of bundles of cable to perform data communication the wired devices are much more costly, especially due to the installation and maintenance. Moreover the wired system is limited to retrofitting the existing light system in buildings. To overcome this installation cost and issues, wireless technology has become a more popular alternative on the demand-side energy management, monitoring and control in buildings. WSN is the enabling technology for building energy control as it is much easier and flexible to install and implement than wired networks.[1]

A lighting control system is proposed that considers both users' preferences and energy conservation. This system senses the location of each user is known via a object detection sensor that is install beside each lighting source. The system also detects local light intensity through LDR and adjust the intensity of installed light source. An additional assumption is that there is no obstacle between whole lighting devices and fixed sensors.[2]

This light control system can control illumination intensity of an LED light based on brightness of surrounding and movement of residents. low cost design is introduced to conserve electrical energy taking daylight illumination into consideration by using LDR. The lighting control system for energy savings in current markets can support on-off and dimming control as managing lighting devices after detecting an object or intensity of illumination, or controlling with time setting. Furthermore, although most existing systems have variable control parameters, it is difficult for users to modify these parameters, so that it is not appropriate to be applied in various places. In addition, although the lighting control system using central management server or sensor network's was implemented recently, it was not commercialized or industrialized, and even the commercialized products were excessively concentrated to the central management server.[3]

\section{SYSTEM ARCHITECTURE}

The following fig.-1 System Architecture shows overall system architecture which describes whole system functionality with the help of four different modules.

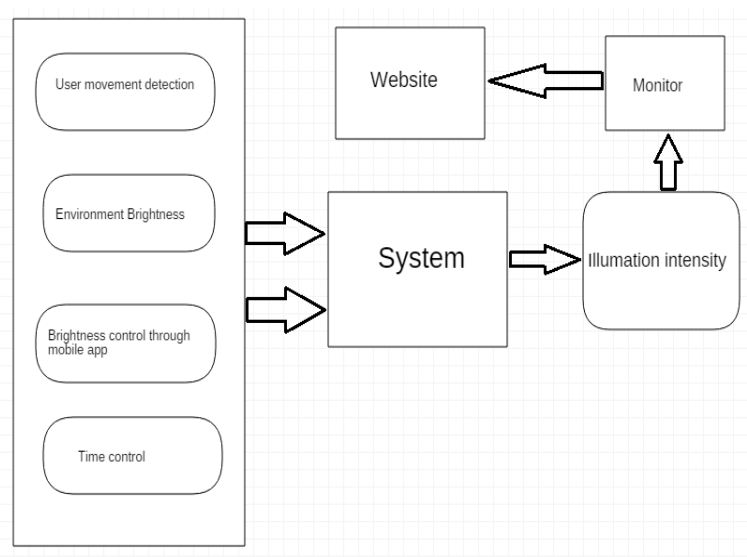

Figure-1 System Architecture

The above figure shows four different system modules namely User Motion Detection, Environmental Brightness, Bright control through Mobile App and Time control. Let's take first module, in this module our objective is to detect the user's entering in the room through PIR sensor. Once the user/object enters in the room the PIR generate some signal this signal acknowledgement to the system which then controls the light by switching ON-OFF the LED's.[4]

In next module we are going to concentrate on ambient light for this purpose we are using LDR. We are going to deploy this LDR at the place from where the ambient light comes inside room. Depending on intensity of ambient light we control intensity of installed LED lighting devices.[5]

The third module describe brightness control through mobile application. In this module we are going to develop one android application. We will install this application on user's mobile phones which will gives ability to user's to control install lighting devices remotely. User can either ON-OFF Led devices and he/she can adjust the intensity of LED devices through this application.[6] 
The last module describe about time control. In this module we are going to apply time control constraint to installed lighting devices in order to restrict this devices to glow with in specific period of time. The time constraint depends on application area this means where we are going to deploy this system.[7]

\section{IV.DEVICES AND DRIVERS}

The following devices we are using to implement our system. These are the electronic components i.e. sensors which sense the data and generate the required output.

\section{- Raspberry Pi-3:}

The Raspberry Pi hardware has evolved through several versions that feature variations in memory capacity and peripheral-device support. It is the hardware that contains its own operating system that used to interfacing with other sensors and cloud.

In this project the Raspberry $\mathrm{Pi}-3$ is the main component through which we can control and monitor the lightning devices. We are used this processor because it is having different features like performance and cost. It is also a high speed processor.

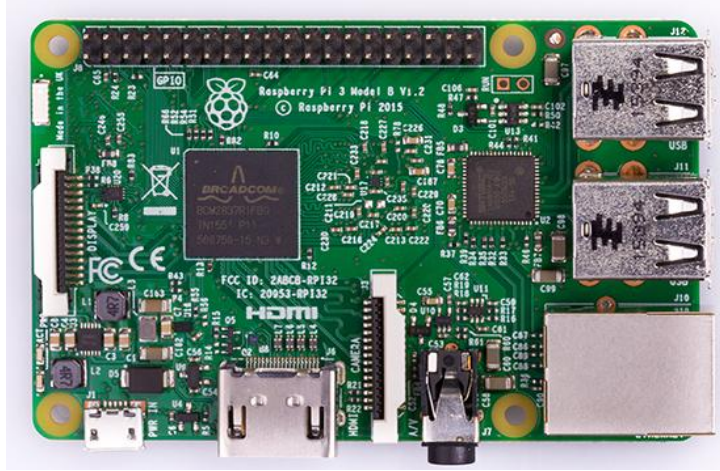

Figure-2 Raspberry Pi-3

\section{- Pyroelectric Infrared Sensor(PIR):}

A passive infrared sensor (PIR sensor) is an electronic sensor that measures infrared (IR) light radiating from objects in its field of view. They are most often used in PIR-based motion detectors. All objects with a temperature above absolute zero emit heat energy in the form of radiation.

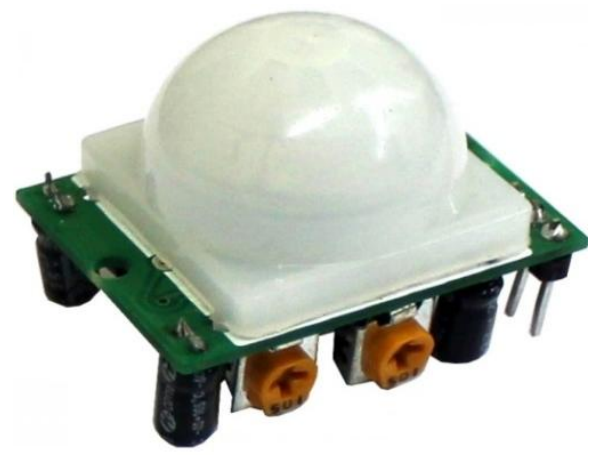

Figure-3 Pyroelectric Infrared Sensor (PIR)
Usually this radiation isn't visible to the human eye because it radiates at infrared wavelengths, but it can be detected by electronic devices designed for such a purpose. We are using PIR for user movement detection through which we can control the light.

\section{- Light Dependent Resistor (LDR):}

A photo-resistor (or light-dependent resistor, LDR, or photocell) is a light-controlled variable resistor. The resistance of a photo-resistor decreases with increasing incident light intensity; in other words, it exhibits photoconductivity. A photo-resistor can be applied in light-sensitive detector circuits, and light- and darkactivated switching circuits. We are using the LDR because to capturing the environmental brightness and control the lighting devices.

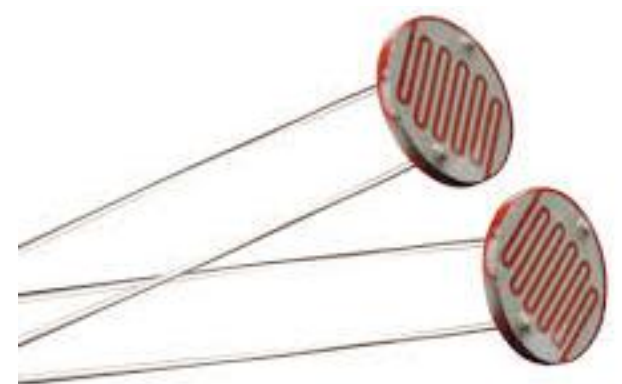

Figure- 4 Light Dependent Resistor (LDR)

\section{CONCLUSION}

Saving energy has become one of the most important issues now a day's. A light accounts larger part of the world's total energy consumption. However, since there are no products considering both energy efficiency and user satisfaction, the existing systems cannot be successfully applied to home and office buildings. The use of a light sensor and a PIR sensor in combination with the Users preferences allows the distributed intelligence to save energy reducing the light intensity. Because many fixtures of LED lights are already placed, this solution is also suitable for retrofitting. Moreover the network is flexible and scalable due to the wireless radio. System results indicate that system outperforms the state-of-the-art with a significant reduction of power consumption and cost for the single and groups of LED lights using the low power, scalable WSN. It has been shown that this system decreases the power consumption in a real life office application by few prototypes are ready to be inserted in a commercial driver to enable wireless capability and distributed control. This system utilizes multi sensors and wireless communication technology in order to control an LED light according to the user's state and the surroundings.

\section{FUTURE SCOPE}

This system is generally developed for saving large amount of energy. This system is having huge scope like 
we used it at College building, Street Light on highways, Home appliances and many others IT Sectors for saving energy.

\section{ACKNOWLEDGMENT}

It gives us great pleasure in presenting the preliminary project report on 'Adaptive Framework for Energy Efficiency and User Satisfaction.' We would like to take this opportunity to thank our internal guide Dr. Pramod Patil for giving us all the help and guidance we needed. We are really grateful to him for his kind support. His valuable suggestions were very helpful. We are also grateful to Prof. Santosh V. Chobe, Head of Computer Engineering Department, Dr. D. Y. Patil Institute of Engineering and Technology, Pimpri for his indispensable support, suggestions. In the end our special thanks to Prof. C. B. Joshi for giving us unending support for the project.

\section{REFERENCES}

[1] Intelligent Household LED Lighting System Considering Energy Efficiency and User Satisfaction' Jinsung Byun, Insung Hong, Byoungjoo Lee, and Sehyun Park, Member, Date 1, February 2013.

[2] Indoor LED Lighting Control System Using WSN Considering User Satisfaction Q. Y. Zhou, Z. L. Zhang, and F. Y. Cui, Date Sep 2014.

[3] Y. K. Tan, T. P. Huynh, and Z. Wang, 'Smart personal sensor network control for energy saving in DC grid powered LED lighting system' IEEE Trans. Smart Grid, vol. 4, no. 2, pp. 669676 , Jun. 2013.

[4] Caicedo and Ashish Pandharipande, 'Distributed Illumination Control With Local Sensing and Actuation in Networked Lighting Systems' IEEE Sensors Journal, Vol. 13, No. 3, March 2013

[5] S. Matta and S. M. Mahmud, 'An intelligent light control system for power saving in Proc. 36th Annu. Conf. IEEE Ind. Electron. Soc. (IECON), Nov. 2010, pp. 33163321.

[6] M. Erol-Kantarci and H. T. Mouftah, Wireless sensor networks for cost efficient residential energy management in the smart grid,' IEEE Trans. Smart Grid, vol. 2, no. 2, pp. 314325, Jun. 2011

[7] Y. Uhm, I. Hong, G. Kim, B. Lee, and S. Park, Design and implementation of power-aware LED light enabler with locationaware adaptive middleware and context-aware user pattern, IEEE Trans. on Consumer Electron., vol. 56, no. 1, pp. 231-239, Feb. 2010.

[8] F. J. Bellido-Outeirino, J. M. Flores-Arias, F. Domingo-Perez, A. Gil-de-Castro, and A. Moreno-Munoz, Building lighting automation through the integration of DALI with wireless sensor networks, IEEE Trans. on Consumer Electron., vol. 58, no. 1, pp. 47-52, Feb. 2012

[9] F. Leccese, 'Remote-Control System of High Efficiency and Intelligent Street Lighting Using a ZigBee Network of Devices and Sensors,' IEEE Trans. on Power Delivery, vol. 28, no. 1, pp. 21-28, Jan. 2013. 